\title{
Efficient Methods for Generating MARS-Like S-Boxes
}

\author{
L. Burnett, G. Carter, E. Dawson, and W. Millan \\ Information Security Research Centre, \\ Queensland University of Technology, \\ GPO Box 2434, Brisbane 4001 \\ Queensland, Australia \\ FAX: +61-7-3221 2384 \\ Email: \{burnett, dawson\}@isrc.qut.edu.au
}

\begin{abstract}
One of the five AES finalists, MARS, makes use of a 9x32 s-box with very specific combinatorial, differential and linear correlation properties. The s-box used in the cipher was selected as the best from a large sample of pseudo randomly generated tables, in a process that took IBM about a week to compute. This paper provides a faster and more effective alternative generation method using heuristic techniques to produce $9 \times 32$ s-boxes with cryptographic properties that are clearly superior to those of the MARS s-box, and typically take less than two hours to produce on a single PC.
\end{abstract}

\section{Introduction}

The Data Encryption Standard [7] has, for the past 25 years, been the US standard for symmetric (shared-key) encryption. In recent years, however, its block and key length have proved to be incapable of providing the levels of security required for applications which utilise shared key encryption. The call for a new standard to replace the Data Encryption Standard for shared-key encryption has been a controversial issue within the cryptographic community for the past two years. The new standard to be known as the Advanced Encryption Standard (AES) [8] has currently been narrowed down to five candidates out of the fifteen initial submissions to the call for AES algorithms in 1997.

The security of a block cipher rests almost entirely on the strength of the components of which it is comprised. These components must not only be secure individually, but must also achieve a much higher level of security when organised together as a cipher system. Substitution boxes (s-boxes) are one of the most important components of a block cipher. They contribute a variety of strengthening properties to the cipher as a whole. Their basic mechanism of allowing bits coming in to an s-box to be replaced with bits going out of an s-box makes them an obvious (and often the only) means of providing nonlinearity to a cipher.

One of the five final candidates for the AES is the MARS cipher [3]. IBM, the designers of the MARS symmetric block cipher, have generated a 9x32 s-box 
which is used in various stages of its computations, both as a 9x32 s-box and as two $8 \times 32$ s-boxes. A large effort was undertaken on the part of the designers to generate an s-box which satisfied the very specific properties outlined in the MARS documentation. This required an approximate program execution time of "about a week". However, as will be shown in Section 3, the MARS s-box does not satisfy all the required properties.

In this paper we present an alternative approach to the generation of MARSlike s-boxes using a heuristic technique known as hill climbing. We discuss the cryptographic properties achieved by hill climbing, and in particular give a comparison between these and the property requirements of the MARS s-box. We show that in order for our hill climbing application to satisfy the requirements of the MARS s-box, the program execution time for generation of an s-box was at most 3.3 hours. The average generation time for a 9x32 MARS-like s-box using our approach was approximately 2 hours. Apart from speed, hill climbing provides individual output functions that have cryptographic properties superior to those of the MARS s-box output functions.

The remainder of this paper is set out as follows: In Section 2 we outline some important fundamentals in s-box theory. In Section 3 we comment on the cryptographic property requirements of the MARS s-box. Section 4 discusses the techniques used by the designers of MARS to generate the $9 \times 32$ s-box used in their computations. We also describe our alternative technique for generating MARS-like s-boxes which satisfy the same requirements imposed by the MARS designers. In Section 5 we discuss some possible variations to our generation technique and relationships between requirements for the MARS s-box and our s-box. Some concluding points are put forward in Section 6, together with some directions for future research in this area.

\section{S-Box Fundamentals}

An $M \mathrm{x} N$ substitution box (s-box) is a mapping from $M$ input bits to $N$ output bits. There are $2^{M}$ inputs and $2^{N}$ possible outputs for an $M \mathrm{x} N$ s-box. A $9 \mathrm{x} 32$ s-box, such as the MARS s-box, has 9 input bits and thus $2^{9}=512$ possible inputs. Each input maps to a 32-bit output word.

S-boxes can also be considered as an ordered set of single output boolean functions. The truth table of a boolean function $f(x)$ is a vector containing $2^{M}$ elements, each element $\in\{0,1\}$. The polarity truth table of a boolean function, denoted $\hat{f}(x)$, is a simple translation from the truth table where every element 0 in the truth table is replaced by the element 1 in the polarity truth table and every element 1 in the truth table is replaced by the element -1 in the polarity truth table. The relationship can be defined as $\hat{f}(x)=1-2 f(x)$.

The hamming distance between two boolean functions $f(x)$ and $g(x)$ is the number of truth table positions in which they are different. The Walsh Hadamard Transform (WHT) of a boolean function, denoted $F(\omega)$, is defined as 
$F(\omega)=\sum_{x} f(x) L_{\omega}(x)$ where $L_{\omega}(x)$ is the linear function selected by $\omega$

and gives a measure of the correlation between a boolean function and the set of all linear functions and their complements. Note that a linear function, $L(x)$ $=\sum a_{i} x_{i}$ with $a_{i} \in\{0,1\}$.

Boolean functions (and therefore s-boxes) are required to exhibit cryptographic properties in order for them to effectively resist certain cryptanalytic attacks. We briefly describe below some of these properties.

A boolean function of $M$ input variables which contains $2^{M-1}$ ones in its truth table is said to be balanced. This property ensures that there is no bias in the truth table. The advantage of using balanced boolean functions is that they cannot be approximated by a constant function. Thus balance is a desirable property to achieve in boolean functions.

The nonlinearity of a boolean function $f(x)$ of $M$ variables is given by

$$
N_{f}=\frac{1}{2} \times\left(2^{M}-W H_{\max }\right)
$$

where $W H_{\max }$ represents the maximum absolute value of the Walsh Hadamard Transform. The nonlinearity of a boolean function is the minimum Hamming distance to the set of all affine (linear) boolean functions. By this definition a boolean function with high nonlinearity cannot be well approximated by a linear function, thus making the function more resistant to linear cryptanalysis. For this reason, nonlinearity is considered to be one of the most important cryptographic properties of boolean functions.

The autocorrelation function, denoted $\hat{r}_{f}(s)$, of $\hat{f}(x)$, the polarity truth table of $f(x)$, can be expressed as

$$
\hat{r}_{f}(s)=\sum_{x} \hat{f}(x) \hat{f}(x \oplus s) .
$$

This cryptographic property provides a measure of the imbalance of all first order derivatives of the boolean function $f(x)$. A boolean function with low autocorrelation makes it more resistant to differential cryptanalysis in that the lower the autocorrelation value, the more difficult it is to approximate the function's first order derivatives.

An $M$-variable function $f(x)$ is said to be $k^{t h}$ order correlation immune, denoted $\mathrm{CI}(k)$, if it is statistically independent of the subset $x_{i_{1}}, x_{i_{2}}, \ldots, x_{i_{k}}$ of input variables where $1 \leq k \leq M$. In terms of security, the output of a correlation immune boolean function, reveals no information about small subsets of input values.

As boolean functions are the building blocks of s-boxes, it is typical to require the same cryptographic properties to be present in s-boxes to improve their strength and ability to resist existing cryptanalytic attacks as well as possible future attacks. 


\section{MARS Property Requirements}

\subsection{MARS Differential Requirements}

The designers of the MARS cipher, in designing their 9x32 s-box, placed particular emphasis on ensuring that their s-box satisfied a number of property requirements. In this subsection, we discuss these requirements with respect to the combinatorial/differential properties of the s-box and also point out where the s-box does not satisfy one of the stated properties.

Note that they have referred to their $9 \times 32$ s-box as $\mathrm{S}[\mathrm{i}], 0 \leq \mathrm{i} \leq 511$. This s-box may be divided into two $8 \times 32$ s-boxes, $\mathrm{S} 0[\mathrm{j}]$ and $\mathrm{S} 1[\mathrm{j}]$, where $0 \leq \mathrm{j} \leq 255$. For ease, we will adopt this notation here also.

\section{Differential Requirements from [3]}

1. S does not contain either the word 0x00000000 (all zeros word) or the word 0xffffffff (all ones word).

2. Every pair of distinct entries in each of the two $8 \times 32$ s-boxes, S0 and S1, differs in at least three out of four bytes. Equivalently, a pair of words from the same $8 \times 32$ s-box may have no more than one byte the same, in the same position.

3. The $9 \times 32$ s-box, $S$, does not contain two entries $S[i]$ and $S[j](i \neq j)$ such that:

i) $\quad \mathrm{S}[\mathrm{i}]=\mathrm{S}[\mathrm{j}]$;

ii) $\quad \mathrm{S}[\mathrm{i}]=\neg \mathrm{S}[\mathrm{j}]$; or

iii) $\quad \mathrm{S}[\mathrm{i}]=-\mathrm{S}[\mathrm{j}]$.

In other words, there are no two entries in $\mathrm{S}$ which are (i) identical; (ii) are complements of each other; and (iii) sum modulo $2^{32}$ to give zero.

4. (i) The xor difference of each distinct pair of entries in $\mathrm{S}$ is unique and (ii) the subtraction difference of each distinct pair of entries in $\mathrm{S}$ is unique.

5. Each distinct pair of entries in $\mathrm{S}$ differs by at least four bits.

An examination of the way the s-box entries of MARS are incorporated into the cipher reveals why requirements $1-5$ are important. An input value selects an s-box entry and this entry is either xored with, added modulo $2^{32}$ to, or subtracted modulo $2^{32}$ from an intermediate value. By excluding the all zero subblock, any xor operation involving an s-box entry and an intermediate value changes the intermediate value. In addition, exclusion of the all one subblock ensures that not all bits of an intermediate value are altered. Requirements 2 and 5 ensure that any two distinct entries in $\mathrm{S}$ are somewhat different at both the byte and bit level. Requirement 3 ensures that the effect of one s-box entry 
cannot be cancelled by another entry from the s-box. Requirement 4 ensures that each possible output "difference" of the s-box is equiprobable, i.e. the Difference Distribution Table is flat.

\subsection{MARS Linear Requirements}

In this subsection, we discuss the linear requirements imposed by the designers of MARS for their 9x32 s-box.

We note that the linear correlation properties of any $M \mathrm{x} N$ s-box can be considered as a $\left(2^{M}\right) \mathrm{x}\left(2^{N}-1\right)$ matrix where the columns are the Walsh-Hadamard transform vectors of the boolean functions formed by xoring all non-empty subsets of the output functions. Thus the linear requirements can be restated as bounds on the values taken in this linear correlation matrix [2]. Since $M$ is large, even calculating this matrix is very expensive, however we may calculate any individual column we like. The MARS linear requirements are all bounds on particular column subsets of this matrix, which can be calculated easily. It should be noted that the vast majority of the s-box linear correlation columns are not considered in any way by the MARS linear correlation requirements.

In addition, there are strict limitations on the values that can be taken for the correlations between boolean functions, and hence also for the values of bias that can occur. The bias values for an $M$-input boolean function can only take rational values that are a multiple of $2^{-M}$. Thus the choice of bias values $\frac{1}{30}$ and $\frac{1}{22}$ in the property requirements needs explanation.

\section{Linear Requirements from [3]}

\section{Parity Bias:}

The parity bias of $\mathrm{S}$ given by $\left|\operatorname{Pr}_{x}[\operatorname{parity}(\mathrm{S}[\mathrm{x}])=0]-\frac{1}{2}\right|$ is to be at most $\frac{1}{32}=0.03125$.

This requirement is a bound on the magnitude of the imbalance of the boolean function formed by xoring all output functions. This property thus affects only one column of the linear correlation matrix, that column being the xor sum of all 32 output boolean functions.

\section{Single-bit Bias:}

The single-bit bias of $\mathrm{S}$ given by $\left|\operatorname{Pr} r_{x}\left[\mathrm{~S}[x]_{i}=0\right]-\frac{1}{2}\right| \forall \mathrm{i}$ is to be at most $\frac{1}{30} \approx 0.03333$.

This requirement places a bound on the magnitude of the imbalance for all of the individual output functions. Thus 32 columns of the linear correlation matrix are affected. 


\section{Two Consecutive Bits Bias:}

The two consecutive bits bias of $\mathrm{S}$ given by $\left|\operatorname{Pr}_{x}\left[\mathrm{~S}[x]_{i} \oplus \mathrm{S}[x]_{i+1}=0\right]-\frac{1}{2}\right|$ $\forall \mathrm{i}$ is to be at most $\frac{1}{30} \approx 0.03333$.

This requirement places a bound on the magnitude of the imbalance of boolean functions formed by the xor of two adjacent outputs. There are 31 of these pairs, hence 31 matrix columns are affected.

\section{Single-bit Correlation}

The single-bit correlation of $\mathrm{S}$ given by $\left|\operatorname{Pr}_{x}\left[\mathrm{~S}[x]_{i}=x_{j}\right]-\frac{1}{2}\right| \forall \mathrm{i}, \mathrm{j}$ is to be minimised. The single-bit correlation bias for the MARS s-box is less than $\frac{1}{22} \approx 0.04545$.

This requirement seeks to minimise the correlation that all output functions have with the individual input bits. This requirement affects $32 \times 9=288$ matrix columns.

In all, only a maximum of 352 matrix columns out of $2^{32}-1$ are considered by the MARS linear requirements. With these same requirements, we are able to show that our heuristic processes are able to generate better properties, much quicker.

\subsection{Satisfaction of MARS Properties}

We shall now discuss the extent to which the MARS s-box was able to achieve the above properties.

\section{MARS S-Box, S comprised of S0 and S1}

S satisfies differential conditions 1,3 and 5 . S0 and S1 both satisfy differential condition 2. S does not satisfy differential condition 4 . In [3], the authors state that S has 130816 distinct xor-differences and 2 x 130816 distinct subtractiondifferences. This is not the case. $\mathrm{S}$ has 130813 distinct xor-differences and 2 x 130808 distinct subtraction-differences as evidenced below. In each equation, the xor/subtraction difference of the indexed words on the left is equal to the xor/subtraction difference of the indexed words on the right.

$\mathrm{S}[27] \oplus \mathrm{S}[292]=\mathrm{S}[101] \oplus \mathrm{S}[360]$
$\mathrm{S}[27] \oplus \mathrm{S}[101]=\mathrm{S}[292] \oplus \mathrm{S}[360]$
$\mathrm{S}[27] \oplus \mathrm{S}[360]=\mathrm{S}[101] \oplus \mathrm{S}[292]$
$\mathrm{S}[13]-\mathrm{S}[138]=\mathrm{S}[364]-\mathrm{S}[297]$
$\mathrm{S}[13]-\mathrm{S}[364]=\mathrm{S}[138]-\mathrm{S}[297]$
$\mathrm{S}[19]-\mathrm{S}[168]=\mathrm{S}[509]-\mathrm{S}[335]$
$\mathrm{S}[19]-\mathrm{S}[509]=\mathrm{S}[168]-\mathrm{S}[335]$
$\mathrm{S}[49]-\mathrm{S}[142]=\mathrm{S}[97]-\mathrm{S}[392]$
$\mathrm{S}[49]-\mathrm{S}[97]=\mathrm{S}[142]-\mathrm{S}[392]$


$\mathrm{S}[333]-\mathrm{S}[131]=\mathrm{S}[211]-\mathrm{S}[348]$

$\mathrm{S}[333]-\mathrm{S}[211]=\mathrm{S}[131]-\mathrm{S}[348]$

The parity bias of $\mathrm{S}$ is $2^{-7}$, as stated in the MARS paper, which is less than the threshold value of $\frac{1}{32}$. The single-bit bias of $\mathrm{S}$ is at most $\approx 0.033203$ which is slightly less than the limit of $\frac{1}{30} \approx 0.033333$. The two consecutive bits bias of $\mathrm{S}$ is at most $\approx 0.032290$ which is less than the bound of $\approx 0.033333$. The maximum single-bit correlation bias of $\mathrm{S}$ is about $0.044922<0.0454545$, as stated in the MARS paper. Thus all linear conditions imposed by the designers of the MARS s-box are satisfied by $\mathrm{S}$.

\section{S-Box Generation Techniques}

\subsection{Summary of MARS S-Box Generation Techniques}

As mentioned earlier, the MARS s-box is a 9x32 s-box containing 512 32-bit entries. The approach taken by the designers of the s-box was to generate the 9x32 s-box by using the well known SHA-1 (Secure Hash Algorithm-1) [9]. SHA1 produces a 160-bit digest comprised of the concatenation of five 32-bit words. The input used for SHA-1 is the value $5 \mathrm{i}|\mathrm{c} 1| \mathrm{c} 2 \mid \mathrm{c} 3$ where $\mathrm{i}=0 . .102$, cj $(\mathrm{j} \in 1,2)$ are the fixed constants

$$
\begin{aligned}
& \mathrm{c} 1=0 \mathrm{xb} 7 \mathrm{e} 15162 \\
& \mathrm{c} 2=0 \mathrm{x} 243 \mathrm{f} 6 \mathrm{a} 88
\end{aligned}
$$

and c3 is allowed to vary until the first eight property requirements are satisfied. The value for $\mathrm{c} 3$ which minimises requirement nine is then the one chosen. Therefore, each entry of the $9 \times 32$ s-box, $\mathrm{S}$ is computed as follows:

$$
\mathrm{S}[5 \mathrm{i}+\mathrm{k}]=\mathrm{SHA}-1(5 \mathrm{i}|\mathrm{c} 1| \mathrm{c} 2 \mid \mathrm{c} 3)_{k}
$$

denoting the kth word of the output of SHA-1 $(\mathrm{k}=0 . .4, \mathrm{i}=0 . .102)$.

The designers started the computational process with c3 $=0$, increasing its value until the resulting s-box was found. Each value of c3 resulted in a 9x32 s-box which was divided into two $8 \times 32$ s-boxes. For each value of c3, the xor sum of distinct pairs in S0 and S1 was checked to see if it contained more than one zero byte. If this was the case, then S[i] was replaced by 3 - S[i] for one of the words $\mathrm{S}[\mathrm{i}]$ in the pair. The new s-box was again tested for the 5 differential requirements and first 3 linear requirements. If this test was passed then the single-bit correlation was calculated. The final fixed constant value of c3 was 0x02917d59. This value was found to best minimize the single-bit correlation.

As stated in [3], the program for generating $\mathrm{S}$ ran for about a week, with the value of c3 increasing to $0 x 02917459=43086937_{10}<2^{26}$. The MARS s-box can be found in [3]. 


\subsection{Summary of our Techniques for Generating MARS-like S-Boxes}

Our approach to generating MARS-like s-boxes is a flexible one which allows for much variation in parameters and heuristic methods used. The particular technique we chose was a heuristic method known as hill climbing [6].

\subsubsection{Hill Climbing}

Making small changes to the truth table of a boolean function produces one of three effects on the WHT of the function - the WHT values can decrease, remain unchanged or increase. In terms of properties such as nonlinearity, this means that the nonlinearity measure of the new boolean function resulting from the change can either become smaller, remain the same or become larger. Hill climbing takes advantage of this effect to optimise cryptographic properties of boolean functions (and thus s-boxes) by retaining a change which has brought about an improvement in a property value, such as nonlinearity. Such an improvement is incremental and consequently explains the analogy with climbing a hill.

Essentially, hill climbing involves the following steps:

1. Measure the property of concern for the original function.

2. Select a pair of elements to complement ensuring that the pair chosen consists of a zero and a one. (This ensures balance is maintained).

3. Measure the property of concern for the new function.

4. If the measure of the property in 3 is 'better' than the measure of the property of the original function, then accept this new function as the original function. If the measure of the property in 3 is worse, then retain the original function.

5. Repeat steps 2, 3 and 4 until a predetermined stopping criteria has been reached.

\subsubsection{General Procedure}

The technique we used to create our s-boxes began with the generation of random single-output balanced boolean functions. Each boolean function was hill climbed to reach a minimum nonlinearity value, a parameter allowed to vary for optimum results. The goal of this approach was to generate a set of 32 balanced boolean functions which not only each achieved the minimum nonlinearity value set by the user, but was also constrained by a maximum imbalance limit between pairs of boolean functions and was further constrained by a maximum deviation limit from $\mathrm{CI}(1)$.

A set of 32 boolean functions achieving these limits comprise a $8 \times 32$ s-box containing 256 words. Pairs of s-boxes of this size were combined to form a $9 \times 32$ s-box. It seemed less complicated to generate $9 \times 32$ s-boxes in this way due to the necessity of satisfying certain requirements placed on the $8 \times 32 \mathrm{~s}-$ boxes individually. The s-box was then checked for the differential and linear 
requirements placed on the MARS s-box. In order to satisfy differential condition 2 , it was necessary to modify a small number of bytes in each of the $8 \times 32 \mathrm{~s}-$ boxes, typically in less than half a dozen entries, and re-checking that condition, particularly for previous pairs of entries. Similarly, the satisfaction of differential requirement 4 involved replacing a small number of entries in the 9x32 s-box. Subsequently, the new s-box was tested for all 9 conditions again. We ensured that the introduction of any replacement entries in the s-box did not destroy the balance property achieved by the initial functions.

\subsection{Experimental Results}

We stated in Section 3 the differential and linear requirements, together with threshold values set by the designers of MARS for their s-box. We shall now discuss the extent to which our s-box was able to achieve these properties.

\section{Our S-Box, SB comprised of Sb1 and Sb2}

Note that $\mathrm{SB}[\mathrm{i}]$ where $\mathrm{i}=0 . .511$ is a $9 \times 32 \mathrm{~s}-\mathrm{box}$, and $\mathrm{Sb} 1[\mathrm{j}]$, Sb2[j] where $\mathrm{j}$ $=0 . .255$ are both $8 \times 32$ s-boxes. SB can be found in APPENDIX $\mathbf{A}$ and also at http://www.isrc.qut.edu.au/papers/2000/AppendixA.txt.

SB satisfies differential conditions 1, 3, 4 and 5. Sb1 and Sb2 both satisfy differential condition 2 of the MARS s-box requirements.

The parity bias of SB is 0.019531 which is less than the threshold value of $\frac{1}{32}$ $=0.03125$. The single-bit bias of $\mathrm{SB}$ is zero. The absence of any single-bit bias in $\mathrm{SB}$ is due to the balance in each of the 32 boolean functions which comprise the s-box. The two consecutive bits bias of SB is at most $\approx 0.024462$ which is less than the bound of $\approx 0.033333$. The maximum single-bit correlation bias of $\mathrm{SB}$ is $0.03125<0.0454545$. Consequently, all linear conditions imposed by the designers of the MARS s-box are satisfied by our example 9x32 s-box, SB.

The achievement of these results depended largely on the three parameters used in our s-box generation program. For our experiments, we typically generated sets of 32 boolean functions with a minimum nonlinearity of 110, although we experimented with parameters above and below this value. A parameter value for minimum nonlinearity at around 108 produced $8 \times 32$ s-boxes in less than 10 minutes, while minimum nonlinearities of 112 for an $8 \times 32$ s-box took about 3 to 4 hours to generate. Our second parameter was a limit on the maximum imbalance between distinct pairs of boolean functions in the set. A typical parameter value for this limit used in our computations was 10. It was desirable to have a low imbalance between pairs of boolean functions which consequently had the effect of reducing the two consecutive bits bias condition imposed by the MARS s-box designers. We also placed a large degree of importance on our third parameter, the maximum deviation from CI(1). By minimizing this parameter value over all boolean functions, we were easily able to produce a single-bit correlation value below the given bound. Typically, we used parameter values such as 16 or 24 to be the maximum allowable deviation from $\mathrm{CI}(1)$ for the 32 boolean functions comprising the $8 \times 32$ s-boxes. 
A combination of hill climbing and appropriate setting of the parameters discussed above allowed us to produce good $8 \times 32$ s-boxes, pairs of which gave us $9 \times 32$ s-boxes. Most of the s-boxes generated by our technique were very close to satisfying the MARS s-box requirements. In fact, for those s-boxes which we successfully generated, bias values, when exceeded, were so by only extremely small margins. The remaining s-boxes which we generated were easily able to satisfy the same conditions that the MARS s-box satisfies.

Based on a heuristic technique approach, we were able to generate a number of MARS-like s-boxes with little effort. In addition, the program execution time, depending on the parameters chosen, varied from approximately 16 minutes to around 3 hours and 20 minutes on a single Pentium II $300 \mathrm{MHz}$ PC. This time frame is a huge improvement on the program running time for the MARS s-box of about a week.

\section{Property Relationships and Technique Variation}

\subsection{Property Relationships}

An s-box comprised of balanced boolean functions clearly possesses no single-bit bias since the number of ones and zeros in the truth table of balanced boolean functions is the same. Our s-box generation procedure began with the generation of a set of 32 balanced boolean functions. In order to satisfy differential requirements 2 and 4 it was necessary to replace a small number of bytes and entries respectively. However, at all times throughout our computations we retained balance in the boolean functions. None of the boolean functions comprising the MARS s-box are balanced. However, their deviation from balance is not large enough to violate the single-bit bias requirement.

Nonlinearity is a very important cryptographic property of single output boolean functions and s-boxes. Higher nonlinearity indicates a reduction in the magnitude of statistical correlations between sets of input bits and sets of output bits. The nonlinearity of an s-box is measured by the magnitude of the largest Walsh Hadamard Transform (WHT) value in the linear correlation matrix. The linear requirements in [3] are concerned solely with balance properties and no requirements on nonlinearity values are given. The nonlinearity of the individual boolean functions in Sb1 and Sb2 ranges from 108 to 112 inclusive, with an average nonlinearity of 110 . The boolean functions comprising the MARS sboxes, S0 and S1, have nonlinearity values ranging from 92 to 109, the most frequent nonlinearity value being 102 .

Although in our s-box generation procedure we have not directly sought to optimise the autocorrelation property, the boolean functions comprising our s-boxes have, in general, displayed low autocorrelation values. A low autocorrelation distribution for an s-box serves to improve its differential properties, in particular, by flattening the Difference Distribution Table. The range of autocorrelation values for the individual boolean functions in our 8x32 s-boxes, Sb1 and $\mathrm{Sb} 2$, was between 48 and 88 , averaging around 56 . We note that the boolean 
functions comprising the MARS s-boxes, S0 and S1, displayed autocorrelation values of between 52 and 88, averaging 64 .

One of the three important parameters set by our code was a limit for the maximum imbalance of the xor sum of distinct pairs of boolean functions. The purpose of this restriction on boolean function possibilities was to reduce the imbalance between pairs. A low level of imbalance between pairs of boolean functions includes the effect of reducing the two consecutive bits bias i.e. the bias between adjacent output boolean functions.

Our requirement for setting a maximum deviation from $\mathrm{CI}(1)$ for the individual boolean functions is identical to the single-bit correlation requirement placed on the MARS s-box. Minimising this measure reduces the magnitude of correlations which exist between individual input and individual output bits of the s-box.

\subsection{Possible Variations on our Techniques}

A great number of variations to our technique for generating MARS-like s-boxes may be adopted as alternative approaches to this task. An obvious generation method would be to apply another useful heuristic technique called the genetic algorithm to randomly generated boolean functions in order to "build" a cryptographically strong 9x32 s-box. Genetic algorithm applications have been very successful in improving cryptographic properties of boolean functions and s-boxes. Indeed, in [5] it was found that a combined genetic algorithm with hill climbing proved to be even more successful in generating boolean functions with good cryptographic properties such as nonlinearity and autocorrelation.

Additional parameters may be included in the code for the generation of a stronger s-box, for example, criteria for strict avalanche and propagation. Varying the parameters used in the generation process allows for a different strength emphasis in the resulting s-box, although the reader should note the existence of conflicting properties which affect each other in a negative way.

Further, it should be noted that only a small subset of the linear correlation matrix is utilised by the linear requirements imposed by the designers of the MARS s-box. However, as a consequence of our parameter choices a larger subset of the linear correlation matrix is utilised in the generation of our s-boxes, thus making greater use of the information contained in this matrix. We believe that an even stronger s-box can be generated if more information from this matrix is incorporated into s-box design. However, it should be noted that to generate the complete linear correlation matrix and analyse it in its entirety is not practical due to the computational effort required for this task.

\section{Conclusions and Future Research}

The designers of the MARS s-box have successfully generated a 9x32 s-box which satisfies all but one of the requirements placed on it relating to differential and linear properties. Their s-box failed to satisfy differential condition 4 , despite 
claims that it did in fact do so. A long search running through values for c3 caused the program to take about a week to produce the final MARS s-box. In this paper we have presented an alternative approach to the generation of MARS-like s-boxes providing satisfaction of all of the requirements which were placed on the MARS s-box. Further, we have shown that by using a combination of random boolean functions, heuristic techniques and appropriate parameters we have gained additional properties such as higher nonlinearity and balance. This approach requires far less effort and compares very favourably to the MARS approach particularly in terms of computation time and ease of generating not only one but a number of MARS-like s-boxes.

Much work is needed to be done in this area in order to conduct an indepth investigation into the ways in which s-boxes with good cryptographic properties may be generated. The desirable properties are not only limited to those of the differential and linear type, even though their importance, stemming from targets of powerful cryptanalytic attacks, is by no means trivial. In the previous section we have endeavoured to outline a few of the variations on our techniques which could be investigated. Work directed towards other optimisation techniques for improving the cryptographic properties of s-boxes may be another worthwhile path for future research in this area. We believe that the strongest s-box will be one which achieves the correct balance between cryptographic properties. It is an open problem to find this balance.

\section{References}

1. E. BIHAM and A. SHAMIR Differential Cryptanalysis of DES-like Cryptosystems Journal of Cryptology, 4:3-72, 1991.

2. J. DAEMEN, R. GOVAETS and J. VANDEWALLE Correlation Matrices Fast Software Encryption, LNCS vol. 1008, pages 275-285, Springer-Verlag, 1994.

3. IBM Corporation MARS - a candidate cipher for AES http://www.research.ibm.com/security/mars.html.

4. M. MATSUI Linear Cryptanalysis Method of DES Cipher Advances in Cryptology - Eurocrypt'93, LNCS vol. 765, pages 386-397, Springer-Verlag, 1993.

5. W. MILLAN, A. CLARK and E. DAWSON An Effective Genetic Algorithm for Finding Highly Nonlinear Boolean Functions International Conference on Information and Communications Security, ICICS '97 Lecture Notes in Computer Science Vol. 1334, pages 149-158, Springer-Verlag, 1997.

6. W. MILLAN, A. CLARK and E. DAWSON Smart Hill Climbing Finds Better Boolean Functions Workshop on Selected Areas of Cryptology, SAC '97, Proceedings, pages 50-63, 1997.

7. NATIONAL INSTITUTE OF STANDARDS AND TECHNOLOGY (NIST) Data Encryption Standard U.S. Department of Commerce FIPS Publication 46, January 1977.

8. NATIONAL INSTITUTE OF STANDARDS AND TECHNOLOGY (NIST) Request for Candidate Algorithm Nominations for the Advanced Encryption Standard (AES) Federal Register Vol. 62 No. 177, pages 48051-48058. 


\section{NATIONAL INSTITUTE OF STANDARDS AND TECHNOLOGY (NIST) Secure Hash Standard NIST FIPS PUB 180, U.S. Department of Commerce, May, 1993.}

\section{APPENDIX A}

An example S-box generated by our techniques using heuristic methods is $\mathrm{SB}[\mathrm{i}]$ below where $\mathrm{i}=0 . .511$ :

\begin{tabular}{|c|c|c|c|c|c|}
\hline $0 \times 657$ ce571 & 0xb2c0a31b & 0xeaaacac0 & & & \\
\hline 0xb6476df8 & $0 \times 5 d 8 b 1 b d b$ & $0 x 3216 b d 0 c$ & 0x87810f0e & 0x8928aab6 & 0x309926d6 \\
\hline 0x86ed7cda & $0 \times 7$ ce28025 & & & & 0xc635a7c2 \\
\hline 0xb12e7967 & xf3c464ce & & $\mathrm{x} 12 \mathrm{fa} 97 \mathrm{fb}$ & & $x d 8 f 7406 d$ \\
\hline & xf60add94 & x3e297a67 & x61ecf4be & & \\
\hline 0xb01c48c9 & & & & & xd3b54059 \\
\hline 0xd1557eb8 & $\mathrm{x} 6 \mathrm{~d} 1 \mathrm{f} 101 \mathrm{c}$ & & & & xd6746be1 \\
\hline $0 \times 5 \mathrm{e} 026256$ & x57f98f80 & & & & bb9e3 \\
\hline & & & & & $8 \mathrm{c} 07$ \\
\hline 0x9e923d96 & & & & & $7 \mathrm{~b} 720$ \\
\hline $0 \times 2444 \mathrm{c} 1 \mathrm{~d} 0$ & & & & & \\
\hline & & & & & $120 \mathrm{e} 38$ \\
\hline 0xd8ea00da & $0 \mathrm{a}$ & & 998 & & $539 a$ \\
\hline & & & & & $2 \mathrm{~d} 2 \mathrm{cc}$ \\
\hline & & & & & 03971 \\
\hline eb50 & $0 \times 02 e$ & & & & \\
\hline & & & & & \\
\hline & & & & & $184 d$ \\
\hline & & & & & \\
\hline & & & & & \\
\hline 558 & 39 & & & & $49 \mathrm{~d}$ \\
\hline & & & & & \\
\hline & & & & & $\mathrm{d} 552$ \\
\hline & & & & & be5 \\
\hline & & & & & ff77 \\
\hline & & & & & $555 \mathrm{c}$ \\
\hline & & & & & \\
\hline & & & & & $24 \mathrm{e} 0$ \\
\hline & & & & & $\mathrm{aaOcf}$ \\
\hline & & & & & \\
\hline & & & & & $6 \mathrm{da} 6$ \\
\hline & & & & & $650 \mathrm{c}$ \\
\hline & & & & & $077 \mathrm{~b}$ \\
\hline & & & & & edc15 \\
\hline & & & & & $\mathrm{dac} 0 \mathrm{e}$ \\
\hline 0xc23faec3 & $0 x a c c a$ & & & & $\mathrm{a} 00 \mathrm{a} 08$ \\
\hline 0x8d1016ed & 0xa61 & 3e626 & $0 \times 5050 \mathrm{a} 261$ & $0 \times 5$ dcb 8660 & 0x7338f3a 9 \\
\hline & & & & & \\
\hline $0 \times 3 e 5 a 7 c b 8$ & 0xf0b1a844 & $0 \times a 4 a 05227$ & $0 \times 62637655$ & $0 x 07 \mathrm{e} 4 \mathrm{e} 409$ & $0 \mathrm{xad} 8 \mathrm{~d} 8 \mathrm{f} 5 \mathrm{c}$ \\
\hline & & & & & \\
\hline 0xdc7e2e02 & 0x0ece $49 \mathrm{~d} 2$ & 0x853351ed & 0xc1687310 & 0x3b92f374 & 0xf2cfa8dc \\
\hline
\end{tabular}




\begin{tabular}{|c|c|c|c|c|c|}
\hline 0x8eb66314 & $\mathrm{x} 27 \mathrm{ccb} 3 \mathrm{f} 6$ & 0xf7c9237e & $0 \mathrm{x} 4850359 \mathrm{c}$ & & \\
\hline 0x9525bed1 & $\mathrm{x} 046 \mathrm{e} 076 \mathrm{~b}$ & $0 \mathrm{xdc} 54 \mathrm{db} 4 \mathrm{c}$ & 0x3bfca3b5 & & \\
\hline 0x558773ab & x28b2ad90 & 0xf6973f75 & 0xaca086bd & xd7cdc737 & $0 \times 56815 \mathrm{e} 7 \mathrm{~d}$ \\
\hline 0x1f0dbc30 & x78ace509 & $0 x a 6 a 0 a 02 c$ & & & 0xee8681ee \\
\hline 0x3b7bb9c7 & $\mathrm{x} 23 \mathrm{ed} 1 \mathrm{bd} 7$ & 0xe26ef6ce & $\mathrm{x} 8 \mathrm{e} 1 \mathrm{f} 20 \mathrm{db}$ & 0x1becd6e8 & $0 \times 972421 \mathrm{a} 6$ \\
\hline 0x37015bdf & xd2fa8b20 & & & & xad43454d \\
\hline 0xa12419d4 & x764b7009 & $276 \mathrm{e}$ & $\mathrm{x} 590 \mathrm{e} 0446$ & 79 & $\mathrm{x} 464 \mathrm{~b} 03 \mathrm{be}$ \\
\hline 0x36ee34c1 & & & & & xa98bc045 \\
\hline & & $121 d$ & & & 13864 \\
\hline $0 x 8456 \mathrm{e} 39 \mathrm{c}$ & & & & bdc 85129 & x29f8a $23 d$ \\
\hline & & & & & \\
\hline $0 x f d 641 \mathrm{dc} 0$ & f29 & $22 \mathrm{e}$ & & & 3597 \\
\hline & & & & & \\
\hline 9d9084 & & & & & $4 \mathrm{~b} 57$ \\
\hline 0x26441e1b & 43 & & & & \\
\hline & & & & & \\
\hline & & & & & \\
\hline & & & & & \\
\hline & & & & & \\
\hline & & & & & \\
\hline & & & & & \\
\hline & & & & & \\
\hline $0 \times 4$ & ef & & & & 390 \\
\hline & & & & & 477 \\
\hline & & & & & \\
\hline 27823 & 88 & & & & \\
\hline & & & & & 807 \\
\hline & & & & & $\mathrm{b} 2 \mathrm{e}$ \\
\hline & & & & & \\
\hline & & & & & ddc \\
\hline & & & & & \\
\hline & & & & & \\
\hline & & & & & 7 ee \\
\hline & & & & & $\mathrm{c} 1 \mathrm{f}$ \\
\hline & & & & & \\
\hline 0xd7668abf & & 9341 & & & $994 \mathrm{~d} 2$ \\
\hline & & & & & \\
\hline 0xc808f572 & $\mathrm{x} 2 \mathrm{~cd} 72112$ & 0xfd3dcd52 & 0x308db7b4 & 9372 & 7 aca3 \\
\hline & & 0x74b5a608 & & & b967 \\
\hline 0x44fa099c & & & & & \\
\hline 0xa1cefd2e & xababb33d & 0xc64f1a49 & & & $0 x b 44 f 067 c$ \\
\hline 0x0bdaa3a6 & x1d09ecb9 & & & & c3ffo \\
\hline 0x02ef76a8 & 0x8d97a9ee & $0 x 0 \mathrm{edc} 95 \mathrm{e} 5$ & & & 0x4674ffed \\
\hline 0xb3966531 & 0x8d7798b8 & 0x6d021a5e & 0xdb86e411 & 0x78618b37 & 0xafe9287c \\
\hline x9bd $274 \mathrm{c}$ & 0xdd $240 \mathrm{~d} 88$ & $0 \times 4412 \mathrm{~d} 92 \mathrm{a}$ & 0x932082c9 & & \\
\hline
\end{tabular}

